\title{
Men at risk of being a mutation carrier for hereditary breast/ovarian cancer: an exploration of attitudes and psychological functioning during genetic testing
}

\author{
Litanja Lodder ${ }^{*, 1}$, Petra G Frets ${ }^{1}$, R Willem Trijsburg ${ }^{1}$, Aad Tibben ${ }^{1,2,3}$, \\ E Johanna Meijers-Heijboer ${ }^{2}$, Hugo J Duivenvoorden ${ }^{1}$, Anja Wagner ${ }^{2}$, \\ Conny A van der Meer ${ }^{2}$, Peter Devilee ${ }^{4,5}$, Cees J Cornelisse ${ }^{5}$ and Martinus F Niermeijer ${ }^{2}$
}

${ }^{1}$ Department of Medical Psychology and Psychotherapy, Erasmus University Rotterdam/Netherlands Institute for Health Sciences, Rotterdam, The Netherlands; ${ }^{2}$ Department of Clinical Genetics, University Hospital Dijkzigt/ Erasmus University Rotterdam, Rotterdam, The Netherlands; ${ }^{3}$ Department of Neurology, Leiden University Medical Center, Leiden, The Netherlands; ${ }^{4}$ Department of Human Genetics, Leiden University Medical Center, Leiden, The Netherlands; ${ }^{5}$ Department of Pathology, Leiden University Medical Center, Leiden, The Netherlands

Males with a BRCA1/BRCA2 mutation are not at greatly increased risk for cancer, whereas their (grand)daughters, and other female relatives who carry the mutation, are. Males from BRCA1/BRCA2 families may opt for genetic testing to confirm whether or not they may have transmitted the mutation to their children and, if so, to inform them at an appropriate age about the genetic risk and its implications. The psychological implications of genetic testing for men at risk of being a BRCA1/BRCA2 mutation carrier have received little attention. We report on 28 men requesting BRCA1 or BRCA2 testing, and their partners. Men were at $25 \%(n=4)$ or $50 \%$ risk $(n=24)$ of being a mutation carrier, the majority with daughters and half of them with daughters aged over 20 years. Levels of psychological distress were assessed several weeks before and after disclosure of the test result. In addition, we investigated the level of intrusive thoughts and feelings about breast and ovarian cancer and the tendency to avoid these. By means of interviews and questionnaires, participants could report on (expected) emotional implications of genetic testing for themselves and their children on experiences with cancer in the family and, on personality trait optimism. Distress levels prior to the result in tested men and their partners were low. Many men and partners expected the test result to affect their children's, but not their own level of problems. Men without daughters and those with an optimistic personality had especially low distress prior to disclosure. Most men reported that they did not actively avoid the issue. Only four of the $\mathbf{2 8}$ men were identified as mutation carriers. High distress after disclosure of the result was reported by one mutation carrier and by three non-mutation carriers. Verbatim transcripts from interviews showed a large variation of psychological reactions in male mutation carriers (eg regarding guilt feelings). Low pre-test distress in males does not necessarily indicate avoidance of the issue. Future studies may indicate which psychological reactions occur in male mutation carriers when the problem becomes more acute, eg when a daughter is found to carry the mutation and/or is diagnosed with breast or ovarian cancer. European Journal of Human Genetics (2001) 9, $492-500$

Keywords: genetic testing; cancer; males; BRCA1/BRCA2; psychological distress

*Correspondence: LN Lodder, Erasmus University Rotterdam,

Department of Medical Psychology and Psychotherapy, P.O. Box 1738,

3000 DR Rotterdam, The Netherlands.

Tel: +31 10408 7988/408 7812; Fax: +31 10408 9420;

E-mail: I.n.lodder@freeler.nl

Received 29 June 2000; revised 3 April 2001; accepted 17 April 2001 


\section{Introduction}

Mutation analyses of breast cancer susceptibility genes (BRCA1 and BRCA2) have become an option for men and women from hereditary breast and/or ovarian cancer (HBOC) families. ${ }^{1,2}$ The inheritance is autosomal dominant, implying that offspring of female and male mutation carriers have a risk of $50 \%$ of inheriting the mutation. Whereas female mutation carriers have a lifetime risk for breast cancer of $56-87 \%$ and for ovarian cancer of $10-$ $60 \%$, risks for cancer in male mutation carriers are not very high. The lifetime risk for breast cancer in male BRCA2 mutation carriers is $6 \%$ and is marginally increased for some other types of cancer in male BRCA1 and BRCA2 mutation carriers. $^{3-5}$

Thus whereas males from HBOC families are not at high risk for cancer, many of them have close female relatives who are affected with or have died from breast or ovarian cancer, and (grand)daughters for which the mutation in the family has profound consequences. The psychological impact of this particular situation for men in HBOC families has received limited attention. Struewing et $a l^{6}$ reported on the anticipated uptake and the expected consequences of genetic testing in 91 women and 49 men from families suspect for BRCA1. ${ }^{6}$ Whereas the anticipated future uptake for genetic testing was high, men were less likely to opt for testing than women. Besides, significantly fewer men than women expected to become depressed or anxious upon being identified as a mutation carrier.

A qualitative study exploring interviews with 22 men from hereditary breast/ovarian cancer families, showed that these men tended to use avoidance of the topic of hereditary cancer as a coping strategy. ${ }^{7}$ Whereas some men stated that they avoided conversations on breast or ovarian cancer in the family, which were generally conducted among female relatives, others reported that they felt excluded from such discussions.

In a previous study from our group, ${ }^{8}$ four men requesting genetic counselling for BRCA/BRCA2 were interviewed by a psychologist. Only one of these men ultimately decided to obtain his test result, after having twice postponed his appointment for bloodsampling. Two men decided to postpone undergoing the test after their first visit, and one did so after having cancelled the appointment for bloodsampling twice. The psychologist's impression was that these men had problems discussing their experiences with cancer in close relatives, and reflecting upon possible unfavourable implications of testing for themselves and their offspring. A lower uptake for genetic testing in males than in females at risk of inheriting a BRCA1/BRCA2 mutation and a higher drop-out rate in follow up interviews was also described in a larger study from the United States. ${ }^{9}$

The present study aims to provide a more systematic analysis of distress in males at risk of carrying a BRCA1/ BRCA2 mutation who applied for genetic testing, and their partners. Symptoms of anxiety and depression in the weeks prior to and following the test result were assessed. The tendency to actively avoid thinking or talking about breast or ovarian cancer in daily life was also assessed. Prior to disclosure of the result, participants reported on their expectations of the emotional impact of the test for themselves and the children. We aimed to establish whether men who reported high levels of distress in the period before disclosure of the result differed from those with low distress with regard to biographical characteristics, experiences with cancer in the family and personality. Low distress was expected to be associated with having no daughters, having no close relatives with breast and/or ovarian cancer and having a general tendency to stay optimistic in difficult times. We used both questionnaire and interview data, the latter giving an in-depth perspective of the male's experiences and feelings, which might be used to illustrate some results from questionnaire data.

\section{Study population and methods \\ Participants}

Between January 1996 and April 1998, 40 men with a 25 or $50 \%$ risk of having inherited a BRCA1 or a BRCA2 gene mutation who requested genetic testing at the Department of Clinical Genetics of the University Hospital Rotterdam were asked to participate in the psychological study, together with their partners. Of these men, 28 (70\%) returned the pre-test questionnaire and participated in the interview. Twenty-five participants had children (Table 1$)$. Ten men (25\%) decided not to participate and two (5\%) were excluded because they did not return the questionnaire after being interviewed. Most of the declining men reported that they felt reluctance towards participating in the interview. The 12 non-respondents/drop-outs did not differ from the study sample with regard to age, marital status, offspring, age of daughters or prior genetic risk to be a mutation carrier $(P>0.05$; $t$-test for independent samples, two-tailed or $\chi^{2}$ test).

The 28 participating men were at risk for being a BRCA1 $(n=24)$ or a BRCA2 $(n=4)$ mutation carrier, and belonged to 18 different HBOC families. The maximum number of men at risk belonging to one family was four. Twenty-seven men had a partner, 24 of whom joined the study. One of the partners was not included because she was not the mother of the children at risk, two other partners declined participation.

\section{Procedures}

The process of BRCA1/BRCA2 mutation analysis, enabling mutation detection for relatives at risk, has been described previously. ${ }^{10}$ Males at risk who were considering genetic testing had one or more pre-test counselling sessions with the clinical geneticist or genetic nurse. They were informed about the genetic, medical and psychosocial implications of testing for themselves and their children. In case the children were young, it was discussed that the parent may face 
Table 1 Characteristics of the study sample and the nonrespondents

\begin{tabular}{llll}
\hline & $\begin{array}{l}\text { Men } \\
\text { at risk } \\
(\mathrm{n}=28)\end{array}$ & $\begin{array}{l}\text { Partners } \\
(\mathrm{n}=23)\end{array}$ & $\begin{array}{l}\text { Non- } \\
\text { respondents } \\
(\mathrm{n}=12)\end{array}$ \\
\hline $\begin{array}{l}\text { Mean age (years) } \\
\quad \text { (range) }\end{array}$ & 47 & 44 & 52 \\
$\begin{array}{l}\text { Marital status } \\
\text { Married/living together }\end{array}$ & $(29-67)$ & $(25-65)$ & $(29-70)$ \\
$\quad$ Unmarried/divorced & $11 \%$ & & $83 \%$ \\
With children & $89 \%$ & & $17 \%$ \\
With daughters & $79 \%$ & & $92 \%$ \\
$\quad$ Older than 20 years & $50 \%$ & & $70 \%$ \\
Education & & & \\
$\quad$ H High school & $32 \%$ & $36 \%$ & \\
$\quad$ High school & $36 \%$ & $50 \%$ & \\
$\quad>$ High school & $32 \%$ & $14 \%$ & \\
Prior risk & & & $100 \%$ \\
$\quad 50 \%$ & $86 \%$ & & - \\
$25 \%$ & $14 \%$ & & \\
\hline
\end{tabular}

problems if, at some point in the future, the child expresses that he/she would rather not have known about having a $50 \%$ risk of being a mutation carrier. After consideration of these implications, if the test applicant still decided for testing, a blood sample was obtained. The appointment for disclosure of the test result took place $6-8$ weeks later. Men and their partners were informed about the availability of psychological support from a clinical psychologist.

The protocol for this study, which was developed on the basis of the protocol of pre-symptomatic testing for Huntington disease, is described elsewhere. ${ }^{10,11}$ The study was introduced by the clinical geneticist or genetic nurse during the intake/blood sampling session. After agreement to participate, the psychologist (LNL) provided more information and questionnaires to be completed at home. The pretest interview usually took place directly after the blood sampling session, but sometimes several weeks later, at the participants' homes. The post-test assessment (including questionnaires and an interview) took place $1-3$ weeks after the test outcome.

\section{Variables}

General distress The Hospital Anxiety and Depression Scale was administered prior to and following disclosure of the result. The questionnaire consists of two subscales of seven items each, assessing the level of anxiety and depression. ${ }^{12,13}$ Each question has four possible answers and the scores for the two subscales range from 0 to 21 . A score of higher than 10 on either subscale is an indication of clinical anxiety or depression, scores from 8 to 10 indicate 'borderline' anxiety or depression. The validity and reliability of the scale are good. ${ }^{14}$

To enable comparison of levels of anxiety and depression with the normal population, the Symptom Checklist was used prior to disclosure of the result; ${ }^{15}$ this questionnaire has norms for a Dutch female and male population. ${ }^{16}$ This scale was also used to register the answers to the question if one had experienced feelings of guilt in the period prior to disclosure.

Intrusion and avoidance The Impact of Event Scale, assessing the impact of a particular distressing experience, was used prior to and following disclosure of the test result. ${ }^{17}$ The 'Intrusion' (seven items) and 'Avoidance' scales (eight items), measured the extent to which subjects became overwhelmed by thoughts and feelings about breast/ovarian cancer and if they had a tendency to avoid these thoughts and feelings. The score for the intrusion scale ranges from 0 to 35 and for the avoidance scale from 0 to 40 . To enable comparison with results from previous studies on genetic testing of our group, similar response categories were used (never, sometimes, often or continuously); ${ }^{8}$ these response categories differed from those of the original Impact of Event Scale.

Biographical and pedigree information Questions on age, marital status, offspring (number, gender and age) and the educational level were included in the pre-test questionnaire. The pedigree was obtained to examine the prior genetic risk of being a mutation carrier ( 25 or $50 \%$ ).

Experiences with the disease in the family In the pre-test interview, men were asked about their experience with breast and ovarian cancer in relatives. The number of known affected relatives, their place in the pedigree (first, second or third degree relatives), the consequences of the disease, and the lowest age of onset were registered.

Reasons for testing and self-reported (expected) consequences of genetic testing In the pre-test interview, men were asked about their main reasons for testing. They were also asked if they had informed their children about their genetic risk and, if not, at what point in time they expected to inform their children in case they became identified as mutation carriers.

An attitude questionnaire, adapted from previous studies from our group, ${ }^{18}$ monitored the expected emotional consequences of either test result. Men and their partners could indicate whether they expected their own or their children's problems to increase if they were found to carry the mutation. Contrasting expectations after exclusion of a mutation were also explored. Response categories were: 'agree', 'do not know', or 'disagree'.

In the interview prior to disclosure of the result, men were asked in a semi-structured way to report on the implications they expected of either test result. They were asked to initially formulate answers of a limited number of words and then to provide examples from everyday experiences to illustrate their brief answers. This part of the interview was audiotaped and literally transcribed. The post-test interview contained a 
similar semi-structured part, in which men were asked what it meant to them that they were found to be a (non-)mutation carrier. These transcripts were used either to find illustrations for results from questionnaire data, or to provide additional information in case questionnaire data were not conclusive enough.

Optimism At pre-test, a Dutch adaptation ${ }^{19}$ of the Life Orientation Test ${ }^{20}$ was used as a measure of dispositional optimism, assessing a positive attitude towards life (eight items, eg 'In uncertain times I usually expect the best'). The frequency of occurrence of such thoughts or feelings could be indicated on a 4-point scale, ranging from 'almost never' to 'nearly always'.

\section{Statistical analyses}

The Statistical Package for Social Sciences (SPSS/PC, release 8.0) was used. To test differences in means and proportions of two subgroups, $t$-tests for independent samples and $\chi^{2}$ tests for categorical data were used. A $P$-value $<0.05$ (two-tailed) was regarded as statistically significant. The relation between predictive variables and the level of distress was estimated by a single linear regression model. Variables significantly related to the level of distress $(P<0.05$, two-tailed), were included in a multiple linear regression model (backward elimination procedure).

\section{Results}

Study sample

Whereas 24 of the 28 males participating in the study had a $50 \%$ risk of inheriting the BRCA1/BRCA2 gene mutation (the others had a risk of 25\%), only four were identified as mutation carriers. Each of these mutation carriers had one daughter, aged between 10 and 18 years. Of the 24 nonmutation carriers, seven did not return their post-test questionnaires; three expressed reluctance towards answering questions on psychological functioning having received a favourable test outcome, and four did not specify their reasons for declining further participation. Thus, post-test results are available for four mutation carriers and partners $(n=4)$ and 17 non-mutation carriers and partners $(n=14)$. Distress levels prior to disclosure did not differ between non-mutation carriers continuing and non-mutation carriers declining further participation in the study ( $t$-test, two-tailed; $P>0.05$ ).

\section{Descriptives prior to disclosure of the result} Experiences with the disease in the family Seventeen of the 28 men had (had) a mother and/or one or more sisters with breast or ovarian cancer; 15 of these men had also lost one or more of these close relatives due to the disease. Ten men were familiar with the disease only in second degree relatives and one had not known any affected female relative. None of the men knew any male relative to be affected with breast cancer.
Reasons for testing All of the 25 men with children wanted to obtain certainty about whether they could have transmitted the mutation to their offspring. Almost half of these men had children aged less than 15 years. If they became identified as mutation carriers all these men intended to postpone informing their children about their possible risks for several years. Two of the 14 men with adult daughters would opt to inform their daughters about BRCA1/2 testing after having received the test outcome. One of the three men without children, and his partner, wished to include the test outcome in their decision as to whether or not to have children. The other two wanted to undergo genetic testing since they knew that the test was available and saw no reason to postpone it.

Expected consequences of genetic testing An increase of problems for their children was expected by 19 of the 25 men with children and half of their partners $(11 / 21)$, in case they would be identified as a mutation carrier (Table 2). Only nine men and two partners expected their own problems to increase after such a test outcome.

\section{Distress levels prior to disclosure of the result in male participants}

Anxiety and depression prior to disclosure of the result On the Hospital Anxiety and Depression Scale men had a mean pre-disclosure score of 3.0 for anxiety (range: $0-9$; SD: 2.8 ) and of 2.4 for depression (range: $0-7$; SD: 2.3 ), both being far below the 'borderline value' of 8 . Borderline or high scores of anxiety were found in $2 / 28$ men (7\%), and none had similar high scores for depression.

The mean pre-test anxiety and depression scores on the Symptom Checklist were slightly below those of a normal male population. The majority of men (23/26) reported that they had not experienced guilt feelings several weeks prior to disclosure of the result.

Intrusion and avoidance prior to disclosure of the result The mean level of intrusion of thoughts and feelings about breast/ovarian cancer was low (2.7; range: 0-13; SD: 3.2) and the mean level of avoidance of such thoughts/ feelings was even lower (1.7; range: $0-12$; SD: 2.9 ); the full

Table 2 Expected consequences of the test outcome

\begin{tabular}{|c|c|c|}
\hline & $\begin{array}{l}\text { Men at risk } \\
\left(\mathrm{n}=25^{*}\right)\end{array}$ & $\begin{array}{l}\text { Partners } \\
\left(\mathrm{n}=23^{*}\right)\end{array}$ \\
\hline \multicolumn{3}{|c|}{ After being identified as a mutation carrier, I expect... } \\
\hline My children's problems to increase & $76 \%$ & $52 \%$ \\
\hline My own problems to increase & $36 \%$ & $9 \%$ \\
\hline \multicolumn{3}{|c|}{ After being identified as a non-mutation carrier, I expect... } \\
\hline My children's problems to decrease & $83 \%$ & $71 \%$ \\
\hline My own problems to decrease & $44 \%$ & $55 \%$ \\
\hline My mood to improve & $33 \%$ & $18 \%$ \\
\hline
\end{tabular}

*For men/partners with children. 
range of scores on these two scales being $0-35$ and $0-40$ respectively. Looking closer at the answers addressing avoidance, it seems that most men do not consciously avoid the topic, eg none reported avoiding people or situations which reminded them of breast/ovarian cancer, and only 4/ 28 affirmed that they 'simply did not want to think about the disease'.

Predictive factors for pre-test distress in men at risk For the men at risk, we used a linear regression analysis to explore whether biographical characteristics, experiences with cancer in first degree relatives, and an optimistic personality were predictive for levels of pre-test anxiety and depression (Hospital Anxiety and Depression Scale). Two predictive variables were found. Men with higher scores on the optimism scale, were significantly less likely to have high levels of pre-test anxiety $(P<0.01)$ and depression $(P<0.03)$ than non-optimistic men. Also, men without daughters $(n=6)$ had significantly lower depression levels $(P<0.03)$ than those with daughters $(n=22)$. The age of the daughters ( $>20$ or $\leqslant 20$ years of age) was not predictive for distress in men.

\section{Descriptives after disclosure of the result}

Anxiety and depression after disclosure of the result Posttest anxiety and depression levels for each of the four mutation carriers and 17 non-mutation carriers are compared to their scores prior to the result (Figures 1 and 2). At posttest, one mutation carrier and three non-mutation carriers had borderline to high levels of anxiety $(\geqslant 8)$; anxiety levels for these men were already elevated at the pre-test assess- ment. One of these non-mutation carriers and the mutation carrier also reported a borderline level of depression at posttest. The depression levels for these two men had changed little since pre-test.

Mean post-test scores for mutation carriers and nonmutation carriers were, respectively, 4.0 and 3.1 for anxiety (SD: 4.9 and 3.2) and 2.5 and 2.2 for depression (SD: 3.8 and 2.7).

Verbatim interview transcripts of male mutation and nonmutation carriers Because of the small number of mutation carriers in the study sample, no statistical comparison was made between their mean distress levels and those of non-mutation carriers. To obtain some insight into the men's feelings concerning the impact of being identified as a (non-)mutation carrier, we used the verbatim transcripts from the interviews held about 2 weeks after the test result. We describe the words these men used when asked to express in a limited number of words, what it meant to them that they were found to be a (non-)mutation carrier, and their illustrations of these words while using examples from everyday experiences.

Post-test verbatim transcripts of the interview were available for the four mutation carriers and for 10 of the 17 non-mutation carriers. With the other seven non-mutation carriers, interviews were held by telephone and therefore no verbatim transcripts are available. This latter group was included after the main data collection period had passed, in a (failed) attempt to increase the number of mutation carriers in the study.

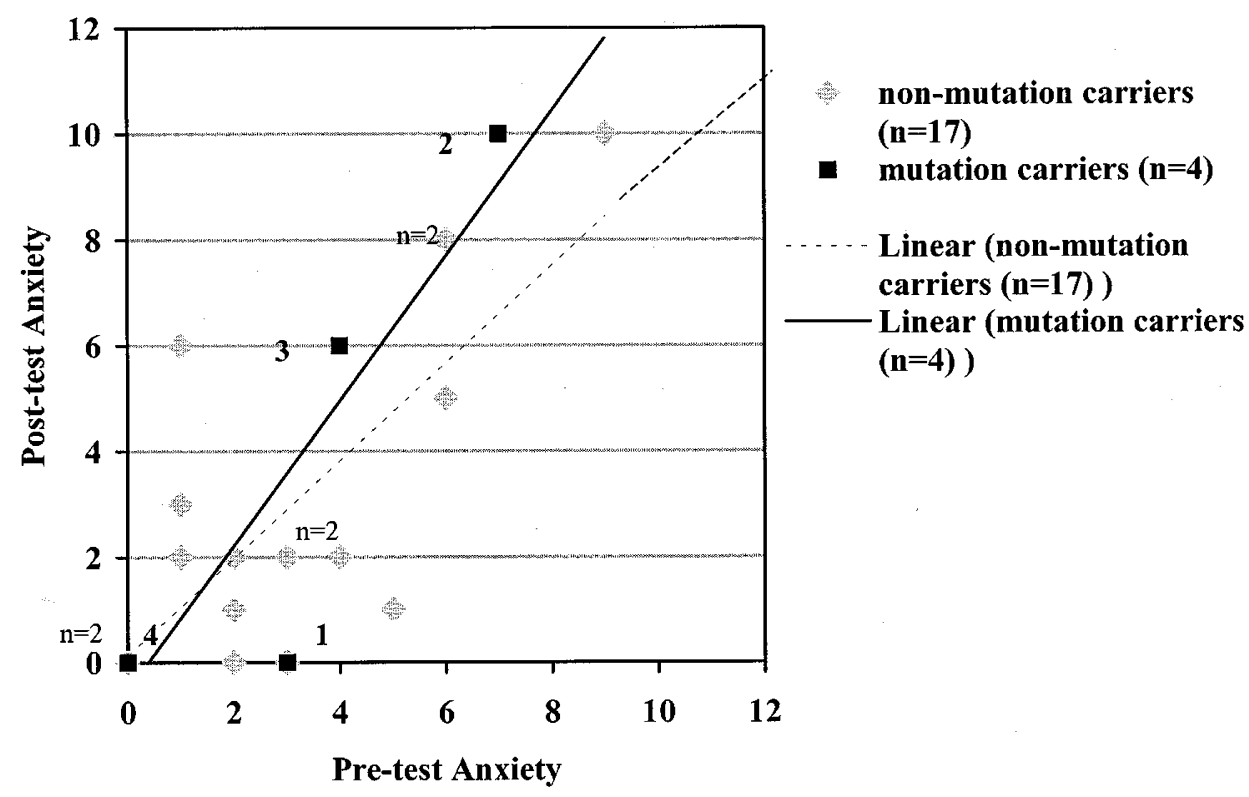

Figure 1 Pre- and post-test anxiety levels in male mutation carriers and non-mutation carriers. One dot can represent more than one subject. The regression line is drawn separately for both groups. 


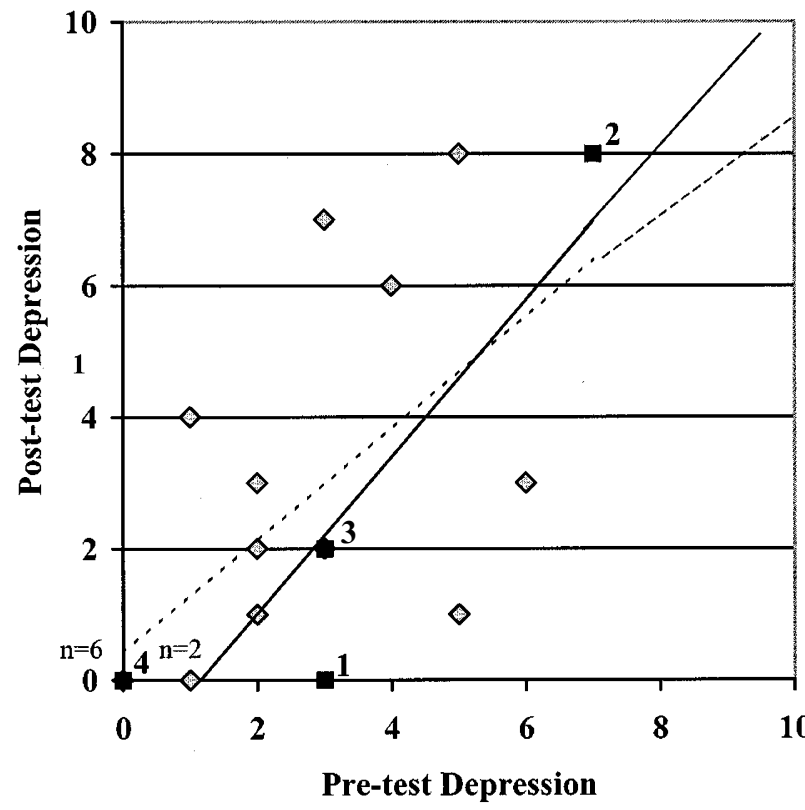

$\diamond \quad$ non-mutation carriers $(n=17)$

- mutation carriers $(n=4)$

-... Linear (non-mutation carriers $(\mathbf{n}=17))$

- Linear (mutation carriers $(\mathrm{n}=4))$

Figure 2 Pre- and post-test depression in male mutation carriers and non-mutation carriers. One dot can represent more than one subject. The regression line is drawn separately for both groups.

Mutation carriers One mutation carrier (with low pre- and post-test distress levels, see ' 1 ' in Figures 1 and 2 ), whose daughter still attends primary school, anwered: [I] 'It is a pity, but I think I am already quite far in the process of accepting the fact that I carry the mutation and that I may have transmitted it to my daughter. I cannot change anything about it, and I do not experience feelings of guilt towards my daughter, neither do I blame my mother for having passed the gene on me. But my perception of things has changed a little since the result. For example, last week my daughter was playing in the garden and I wanted to tell her to come in, but then I let her play a little longer, because she was having so much fun. While I was watching her play I was conscious of the fact that she may have to face difficult times in the future. As a father I want to give her a life as pleasant as possible of course, and now I have the feeling that something has come in between. I think I will become less conscious about this after some time, because it will take many years before my daughter may get the test. In that period of time, a lot can change in the medical world and, besides, she may have a good test result.' Feelings of guilt towards the daughter at risk were explicitly indicated by one mutation carrier (with moderate to high pre- and post-test distress levels, see ' 2 ' in Figures 1 and 2), who planned to postpone informing his adolescent daughter for several years. He and his wife wanted their daughter to live the remaining years of her adolescence without the burden of her elevated risk for cancer. [II]... 'I feel guilty and insecure. Guilty, because if everything had been all right in my body, my children would not have had any problem. And although I know that I cannot help it, it gives me a very bad feeling. Besides, the insecurity I feel is hard to live with. In the first place the insecurity of "does she have this gene, or not". I sometimes have the fantasy of secretly taking some of her blood and having it tested. Secondly, I feel uncertain about what will happen in the future. If my child develops cancer she will be an adult person, but she is still my daughter .'

Two mutation carriers each had one daughter who had just finished high school. One of these daughters wanted to postpone the test until her life became more settled. Her father (who had a moderately high post-test anxiety score, see ' 3 ' in Figure 1) described her as a worrying type of person, and answered: [III]... 'From now on, it will always be in my mind that my daughter may have inherited the gene, unless she undergoes the test and receives a favourable test outcome. A lot of things will remind me of it, for example something on TV or if there is a campaign by the cancer society.' The daughter of the other mutation carrier had undergone the test herself several weeks after she was informed about her father's test outcome. In the week after his own test outcome, her father (who reported an absence of feelings of distress both at pre- and post-test, see ' 4 ' in Figures 1 and 2) said: [IV] ... 'That I am found to be a mutation carrier does not mean anything to me at this moment. This may change if my daughter is also found to be a mutation carrier. On the one hand it may come closer then but, on the other hand, she has not yet reached the age at which it becomes relevant for her. At this moment it simply is not an important issue in my life.' 
Non-mutation carriers Relief about the test result was reported by nine of the 10 non-mutation carriers who were seen in a face-to-face interview $1-2$ weeks after disclosure of the result. One non-mutation carrier said that the result had not positively or negatively affected his feelings. Seven of the 10 men reported that hereditary cancer in the family still affected their lives, because they had relatives who were identified as mutation carriers and/or relatives with breast or ovarian cancer or who died from the disease. None of the non-mutation carriers reported feelings of guilt towards these relatives in their answers. The following quote is from a man who seemed to succeed in separating the grief he felt about deceased female relatives, from the gratefulness he experienced about his own favourable test outcome: [V] ... 'In the past 15 years I have seen all my female relatives, my mother, my sisters, a number of cousins and aunts die. That caused a lot of pain, depending on how close these relatives had been to me, and I feel very grateful that the test has shown that my children and my brother's children do not have to suffer from this black history in our family.' ...

\section{Anxiety and depression in partners of male participants} before and after disclosure of the test result

At pre-test, scores on the Hospital Anxiety and Depression Scale of the partners did not differ much from those of the men in the study. The mean pre-disclosure score was 3.9 for the anxiety scale (range: $0-10 ;$ SD: 2.9) and 1.8 for the depression scale (range: $0-8$; SD: 2.2 ). Borderline anxiety was found in two of the 23 partners (8\%), one of whom also had a borderline depression level. Mean anxiety and depression scores on the Symptom Checklist were somewhat lower than those of a normal female population.

Borderline and/or clinical anxiety levels at post-test, were reported by one of the four partners of mutation carriers. Two of the 14 partners of non-mutation carriers reported similar high anxiety levels, one of whom had reported high anxiety also at pre-test; the latter woman was the only partner with a borderline to clinical level of depression at both pre- and post-test.

\section{Discussion}

\section{Distress in men at risk and their partners prior to disclosure}

Distress levels were explored in 28 men at risk of having inherited a BRCA1/2 mutation (and in their partners) wanting to undergo genetic testing primarily to know about risks for (future) offspring. The majority of the men, and their partners, had low distress levels in the weeks prior to receiving the test result. It should be noted, however, that the study sample was small and not randomly selected. Firstly, 30\% of the men ( $n=12)$ resigned from participation in the study; the biographical characteristics of these nonrespondents did not differ from those participating, but we do not know their levels of distress. Secondly, about half the participating men had one or more male relatives participating in the study, which implies that the sample was not 'statistically independent'. Because of these sample-related restrictions, only cautious interpretations are possible.

The majority of males not only reported low general distress at pre-test, but also low levels of intrusive thoughts/ feelings about breast and/or ovarian cancer. They did not report to be actively avoiding such thoughts/feelings or situations reminding them of the disease. It might be that possible negative implications of testing are perceived as distant. Prior to disclosure of the result, it is not yet known whether one is a mutation carrier and, if so, it is not certain whether (grand)daughters are also mutation carriers. Accordingly, men without daughters had significantly less distress than those with daughters. The impression that men were postponing worrying about the issue until after the result, is also reflected in the result that men with daughters aged over 20 years, whom they would soon inform about the result, were not more distressed than those with young daughters, who could be informed about the result at a much later date.

Considering the latter findings, it is understandable that optimistic test applicants may have felt less distress prior to disclosure than less optimistic test applicants, the former being especially successful in postponing worrying until negative consequences of testing become incontrovertible. For example, one man with a low distress and a high optimism score, stated: [VI] ... 'If I carry this mutation with me I would worry about my daughters, but I am not seeing things too pessimistically, because in five years time they might have found a cure for cancer.' Another man, with a high distress and a low optimism score said: [VII] ... 'If I am found to be a mutation carrier, what future misery will my children have to face, and how much will they have to suffer? I do not assume efficient new treatments to be developed at that time, but I might be doomwatching' ...

In case they became identified as a mutation carrier, three quarters of the men at risk who had children, and half of the partners, expected an increase of problems for their children. Interestingly, half of these men and more than three quarters of these partners reported that this would have no consequences for their own emotional well-being. An explanation for this result in male participants might be twofold. Firstly, men might wish to ignore that their own problems would increase, because they feel that the consequences of being a mutation carrier are far more severe for their daughters. They might perceive themselves as irresponsible or weak if they admit having difficulty themselves. Secondly, most males did not seem to have experienced guilt feelings about the possibility of an increase of problems for their children.

Reporting an absence of guilt feelings prior to disclosure of the result might be because at that moment, men do not yet know whether they could have transmitted the mutation. On the other hand, the impression gained from the questions in 
the interview about the expected impact of being found to be a mutation carrier was that many men seem to be aware that they cannot be blamed for possibly having transmitted the mutation to their children. In literature about guilt, ${ }^{21}$ guilt feelings are characterised by the perception that one is responsible for a bad situation. Individuals feeling guilty tend to use expressions starting with 'had I only...' or 'how could I...'. Feelings of guilt are called irrational if one was not responsible for the outcome, eg if one survives a plane crash or if one has a child with a serious disease. ${ }^{21}$ It is precisely this issue of responsibility which is ambivalent for men at risk for carrying a BRCA1/BRCA2 gene mutation. On the one hand, these men already had children before they knew about the genetic nature of cancer in their family; on the other hand, they may feel responsible because if their children are found to be mutation carriers, they would be the ones who transmitted the mutation. This ambivalence is clearly illustrated by the only test applicant who expressed feeling guilty about the chance of having transmitted the mutation to his daughter: [VIII] ... 'I would feel very miserable about my daughter, if I have inherited the mutation. I would not say that I can be blamed for this, it is due to coincidence, but I find it difficult to accept that this gene comes from my body. It is not that I . . . , yes I actually do, I do feel guilty about this.' This quote clearly shows the complexity of feelings of guilt, and the difficulty to verbalise such feelings. The difficulty to identify painful feelings of guilt may be another reason why some of those who expressed feeling sorry for their children, did not report feeling guilty towards them.

\section{Psychological implications of the test outcome}

Each of the four men who were identified as mutation carriers, seemed to have different levels of concern about their daughters. Two mutation carriers reported not having felt distressed in the weeks following the result, whereas the other two had moderate to high levels of distress. Although the latter two men appeared not to have achieved the level of certainty from testing that they had hoped for, they did not regret having undergone testing. However, the small number of mutation carriers in the present study limits generalisations about the incidence of clinical levels of psychological distress or regrets about having the test done. As long as no data on larger samples are available, genetic counselling should address the fact that feelings of uncertainty about implications for the children may remain or become even stronger if one is identified as a mutation carrier.

About one third of the non-mutation carriers declined to participate in the post-test assessment, some of whom explained they saw no value in further participation because of their favourable test outcome. A previous study also reported a tendency in males from BRCA1/BRCA2 families to cancel appointments for interviews. ${ }^{9}$ The present study does not answer the question whether subjects who withdrew did so to avoid psychological discomfort, or because of a reluctance to continue participating in a process that was no longer a major issue in their lives.

Of the non-mutation carriers who completed the post-test assessment, the majority reported relief about their test outcome. Some were still confronted with the implications of hereditary cancer for female relatives, but none reported feeling guilty about their own favourable test outcome. The explanation for this finding may be similar to the explanation we gave for the low prevalence of guilt towards children at risk. Again, there is no question of responsibility or control in case of relatives being identified as mutation carriers or developing cancer. $^{21}$ Besides, some men may have had difficulty in labeling their feelings of discomfort in terms of guilt.

\section{Concluding remarks}

We conclude that the majority of the men at risk and their partners did not have elevated levels of anxiety and depression in the weeks prior to and following the test result, nor did they report to actively use avoidance to prevent becoming distressed. Whereas participants were concerned about the implications of testing for their offspring, the majority did not need psychological support. The interviews gave the impression that many men perceived the implications from an unfavourable test outcome as distant, and felt that they are not to be blamed for the possibility of having passed the mutation to their offspring. This situation could change drastically, however, if daughters have to undergo invasive prophylactic surgery or become diagnosed with cancer. The difference in emotional impact of such a threatening event and the situation of men in the present study, is clearly summarised by the following quote: [IX] ... 'If I had a daughter affected by cancer, this would affect me twice as badly if this was due to me carrying a gene mutation. I think that as long as things are going fine, you may very well continue to feel all right, but as soon as a daughter becomes ill, you will be overwhelmed by devastating feelings.'

\section{Acknowledgments}

The authors are indebted to Dr DIJ Halley and Dr AMW van den Ouweland, involved in mutation analysis at the Department of Clinical Genetics, Erasmus University Rotterdam.

\section{References}

1 Miki Y, Swensen J, Shattuck-Eidens D et al: A strong candidate for the breast and ovarian cancer susceptibility gene BRCA1. Science 1994; 266: 66-71.

2 Wooster R, Bignell G, Lancaster J et al: Identification of the breast cancer susceptibility gene BRCA2. Nature 1995; 378: $789-792$.

3 Thorlacius S, Struewing JP, Hartge P et al: Population-based study of risk of breast cancer in carriers of BRCA2 mutation. Lancet 1998; 352: $1337-1339$. 
4 The Breast Cancer Linkage Consortium. Cancer risks in BRCA2 mutation carriers. J Natl Cancer Inst 1999; 91: 1310-1316.

5 Ford D, Easton DF, Bishop DT, Narod SA, Goldgar DE. Risks of cancer in BRCA1-mutation carriers. Breast Cancer Linkage Consortium. Lancet 1994; 343: 692-695.

6 Struewing JP, Lerman C, Kase RG, Giambarresi TR, Tucker MA. Anticipated uptake and impact of genetic testing in hereditary breast and ovarian cancer families. Cancer Epidemiol Biomarkers Prev 1995; 4: 169-173.

7 McAllister MF, Evans DG, Ormiston W, Daly P. Men in breast cancer families: a preliminary qualitative study of awareness and experience. J Med Genet 1998; 35: $739-744$.

8 DudokdeWit AC, Tibben A, Frets PG, Meijers-Heijboer EJ, Devilee P, Niermeijer MF. Males at-risk for the BRCA1-gene, the psychological impact. Psycho-oncology 1996; 5: 251-257.

9 Lerman C, Hughes C, Lemon SJ et al: What you don't know can hurt you: adverse psychologic effects in members of BRCA1linked and BRCA2-linked families who decline genetic testing [see comments]. J Clin Oncol 1998; 16: 1650-1654.

10 Lodder LN, Frets PG, Trijsburg RW et al: Presymptomatic testing for BRCA1 and BRCA2: How distressing are the pre-test weeks? J Med Gen 1999; 36: 906-913.

11 Tibben A, Timman R, Bannink EC, Duivenvoorden HJ. Threeyear follow-up after presymptomatic testing for Huntington's disease in tested individuals and partners. Health Psychol 1997; 16: $20-35$.

12 Zigmond AS, Snaith RP. The Hospital Anxiety and Depression Scale. Acta Psychiat Scand 1983; 67: 361-370.

13 Channer KS, Papouchado M, James MA, Rees JR. Anxiety and depression in patients with chest pain referred for exercise testing. Lancet $1985 ; 2: 820-823$.
14 Spinhoven PH, Ormel J, Sloekers PPA, Kempen GIJM. A validation study of the Hospital Anxiety and Depression Scale (HADS) in different groups of Dutch subjects. Psychol Med 1997; 27: $363-370$.

15 Derogatis LR, Rickels K, Rock AF. The SCL-90 and the MMPI: A step in the validation of a new self-report scale. $\mathrm{Br} J$ Psychiatry 1976; 128: 280-289.

16 Arrindell WA, Ettema H. Dimensional structure, reliability and validity of the Dutch version of the Symptom Checklist (SCL90): Data based on a phobic and a "normal" population. Nederlands Tijdschrift voor de Psychologie en Haar Grensgebieden 1981; 36: 77-108

17 Horowitz MJ, Wilner N, Alvarez W. Impact of Event Scale: A measure of subjective stress. Psychoso Med 1979; 41: 209-218.

18 Tibben A, Frets PG, van de Kamp JJ et al: Presymptomatic DNAtesting for Huntington disease: pretest attitudes and expectations of applicants and their partners in the Dutch program. Am J Med Genet 1993; 48: 10-16.

19 Bleiker EM, van der Ploeg HM, Hendriks JH, Ader HJ. Personality factors and breast cancer development: a prospective longitudinal study. J Natl Cancer Inst 1996; 88: 1478-1482.

20 Scheier MF, Carver CS. Optimism, coping, and health: Assessment and implications of generalized outcome expectancies. Health Psychol 1985; 4: 219-247.

21 Madow L. Guilt: How to recognize and cope with it. Northvale: Jason Aronson inc., 1988. 